\title{
Modeling Channel Occupancy Times for Voice Traffic in Cellular Networks
}

\author{
Emre A. Yavuz, and Victor C. M. Leung \\ Department of Electrical and Computer Engineering \\ The University of British Columbia, Vancouver, BC, Canada V6T 1Z4 \\ Email: \{emrey and vleung\} @ece.ubc.ca
}

\begin{abstract}
Call holding times in telephony networks are commonly approximated by exponential distributions to facilitate traffic engineering. However, for traffic engineering of cellular networks, channel occupancy times need to be modeled instead to facilitate analytical modeling or to feed network simulations. In this paper, we classify channel occupancy times and present an empirical study based on data obtained from a real cellular network to determine which probability distribution functions can approximate them better. The results are environment dependent, but no assumptions that can be influential are made, as opposed to previous analytical and simulation studies which results are highly dependent on the assumptions made by the authors. We show that all types of channel occupancy times can be approximated by lognormal distribution. For stationary users, channel occupancy times are commonly approximated by exponential distribution due to its tractability, assuming that cell residence times are also exponentially distributed. However, we show that lognormal distribution fits much better to both channel occupancy and call holding times regardless of whether users are stationary or mobile.
\end{abstract}

\section{INTRODUCTION}

Packet-switched services are gradually integrated with the conventional circuit-switched services such as voice to accommodate network applications that range from text-based utilities to video applications. The advantage of having packet-switched data services overlaid on circuit-switched technology over the same air interface is the utilization of excess network capacity available in each cell. Statistical multiplexing is used to transmit the data packets over the radio interface to overcome the scarcity of system resources and provide a QoS level comparable to that of circuit-switched services. The statistical multiplexing gain arises from the talk spurt to silence ratio found in speech which makes it possible to multiplex more than one service on to the same radio channel. However accurate voice traffic statistics are needed to understand the length and frequency distributions of idle periods of voice channels to design an efficient network.

Traffic statistics are also useful for network traffic modeling, performance evaluation, billing, optimization and allocation of safety buffers. Call holding and channel occupancy times are two of these traffic statistics, which determine performance metrics such as call blocking/dropping probabilities in cellular networks. Call holding time is defined

This work was supported by a grant from Bell Canada under the Bell University Laboratories program, and by the Canadian Natural Sciences and Engineering Research Council under grant STPGP 269872-03. as the duration of requested call connection which corresponds to holding time for a wired phone call or session time in a computer system, whereas channel occupancy time is defined as the time that a mobile occupies a channel within a cell during its residence in the cell. Call holding times are generally assumed to be exponentially distributed when network traffic is approximated. In [1], Hong and Rappaport proposed a traffic model for cellular mobile radio telephone systems and showed that channel occupancy time distribution can be approximated by exponential distribution if call holding times are also distributed exponentially. This assumption has been used extensively in the literature due to its tractability to obtain analytical results when evaluating cellular networks using Markov models. Ramjee et al. [2], Fang and Zhang [3], Naghshineh and Schwartz [4], Gersht and Lee [5], Borst and Mitra [6] and Yavuz and Leung [7] studied performance of various call admission control schemes using one dimensional Markov chain models assuming that channel occupancy times are exponentially distributed. Rappaport in [8] and with Monte in [9] developed multidimensional models based on the same assumption to obtain call blocking probabilities. However simulation studies and field data have shown that these assumptions are not perpetually valid. Guerin [10] used a simulation model to show that channel occupancy time distribution displays a rather poor agreement with the exponential fitting for mobile users with low changes in rate of movement and direction. Using real data from a cellular network, Jedrzycki and Leung [11] showed that the lognormal distribution fits the channel occupancy time data much better that the commonly used exponential distribution. In [12] and [13], Fang et al. demonstrated that channel occupancy times in a cellular network depend not only on call holding times but also on users' mobility which can be characterized by the cell residence time distribution. The authors showed in [14] that channel occupancy time is exponentially distributed if and only if cell residence time is exponentially distributed. Yet it is also observed in the same study that in general channel occupancy time statistics is well represented by the exponential distribution when the mobility is low. In [15], Barcelo and Jordan analyzed a cellular network based on a fully empirical approach and observed that channel occupancy time is less spread out than if the exponential distribution was assumed.

In this paper, we analyze real traffic data obtained from a number of cell sites in Bell Mobility's cellular network. Similar to the empirical study presented in [11], we obtain the probability distribution for channel occupancy times; however we further classify them according to their occupancy types to 
perform goodness-of-fit tests for each types of channel occupancy times and users. In order to facilitate this classification, we briefly review the life cycle of a typical cellular call first.

In a cellular network, the service area is covered by base stations whose radio coverage areas define the corresponding cells. Each base station serves a set of users. When a new call is originated by a user, one of the available channels assigned to a base station is used for communication. If a channel can be assigned to a call, it will be kept until the call is completed or the user moves out of the corresponding cell. When the user moves into a new cell while holding an active call, a new channel needs to be acquired in the new cell using a "handoff procedure." We name the amount of time that a call occupies any channel during its holding time total regardless of being started in a cell and completed in the same cell or handed off to another. We classify channel occupancy times as follows based on the occupancy type characteristics:

- new2same: channels occupied by calls that are started and completed in the same cell.

- new2ho: channels occupied by calls that are started in a cell but handed off to a neighboring cell.

- new2sameorho: channels occupied by calls that are started in a cell and either completed in the same cell or handed off to a neighboring cell. This type of channel occupancy is very important for Markov chain modeling and referred as "new calls" in the models.

- ho2same: channels occupied by calls that are handed off to a cell and completed in that cell.

- ho2ho: channels occupied by calls that are handed off to a cell but handed off again before completed.

- ho2sameorho: channels occupied by calls that are handed off to a cell and either completed in that cell or handed off again before completed. This type of channel occupancy is very important for Markov chain modeling and referred as "handoff calls" in the models.

The characteristics of various types of channel occupancy times are useful for providing sufficiently representative channel occupancy time statistics when developing analytical models and feeding simulations with realistic traffic statistics to obtain network performance metrics. This paper is organized as follows. In Section II, we explain the data analysis method that we use to obtain the statistics for various types of channel occupancy times from the measurement data, and discuss the system related anomalies that we observe. Section III describes the statistical tools, parameter estimation techniques and goodness-of-fit tests utilized in the study. In Section IV, we present the statistical results obtained from the goodness-of-fit tests performed for each aforementioned type of channel occupancy times along with the observed data histograms and fitted distributions. We provide the statistical results obtained from the goodness-of-fit tests performed for channel occupancy and call holding time distributions for stationary and mobile users. We conclude the paper in Section V.

\section{DATA ANALYSIS AND SySTEM-RELATED ANOMALIES}

In this paper, we analyze cellular call data obtained from a CDMA system deployed by Bell Mobility in Ontario, Canada. In Bell's CDMA system a call can be in up to 6 way soft/softer handoff at anytime. However, call handoffs have been modeled differently in the literature in traditional mathematical and simulation network models developed for evaluating performance in cellular networks: a call is traditionally assumed to communicate via one primary sector at any given time unless it is in handoff. In the empirical data set, we obtain the values of $E_{\mathrm{c}} / I_{\mathrm{o}}$, ratio of the pilot signal energy to the total power in the channel, for each active call to determine a call's primary sector. For a call at any given time, we take the sector which the call has the highest $E_{\mathrm{c}} / I_{\mathrm{o}}$ value in its active set as the primary sector of that call for that particular time.

We assume that handoffs are technology independent which happen at the equal power boundaries. We detect the committed handoffs by observing a call's primary sector being replaced by other sectors that have the highest $E_{c} / I_{\mathrm{o}}$ value in the corresponding call's active set at the time of observation. We take an entirely empirical approach in this work based on true data collected from actual working systems. However unlike analytical and simulation approaches, the empirical approach depends on the environment and therefore may contain system related anomalies. The results presented in this paper might have been different if taken in a different place, time, etc. We believe that different approaches are complementary and have their advantages or disadvantages depending on the specific application. Figs. 1 to 7 depict the distributions of measured channel occupancy times classified as new2same, new2ho, new2sameorho, ho2same, ho2ho, ho2sameorho and total respectively. Fitted probability distributions are superimposed. Upon close inspection of the distributions given in Figs. 1 to 7 , we observe two sorts of anomalies: unusually high number of short channel occupancy times and spikes. The unexpected behavior of channel occupancy times classified as ho2ho (see Fig. 5) and ho2sameorho (see Fig. 6) are due to the "pilot pollution" in the data set since several pilot signals are observed to be close to the "add-drop" thresholds and thus come in and get out of their active sets frequently (i.e., no dominant primary sector is identified). The unexpected behavior of the channel occupancy times classified as new2same (see Fig. 1) and new2sameorho (see Fig. 3) and total (see Fig. 7) is similar to what is observed by Bolotin in [16]. The author categorized the observed short channel occupancy times into four different classes in order of increasing average times [16]: various abandonment before the connection is established; outgoing calls that encounter busy condition and being abandoned by the caller; outgoing calls that encounter no answer condition and being abandoned by the caller and outgoing calls that encounter a busy or no answer condition and therefore followed by a voice mail message left by the caller.

A second type of anomaly is the spikes that we observe within channel occupancy time samples classified as new2same around the $16^{\text {th }}$ second and ho2ho around the $4^{\text {th }}$ second. The former can be explained due to calls that encounter no answer and therefore go to voice mail while the latter can be explained due to "immediate handoff candidacy" [11]. The spikes observed around the $16^{\text {th }}$ second samples classified as 


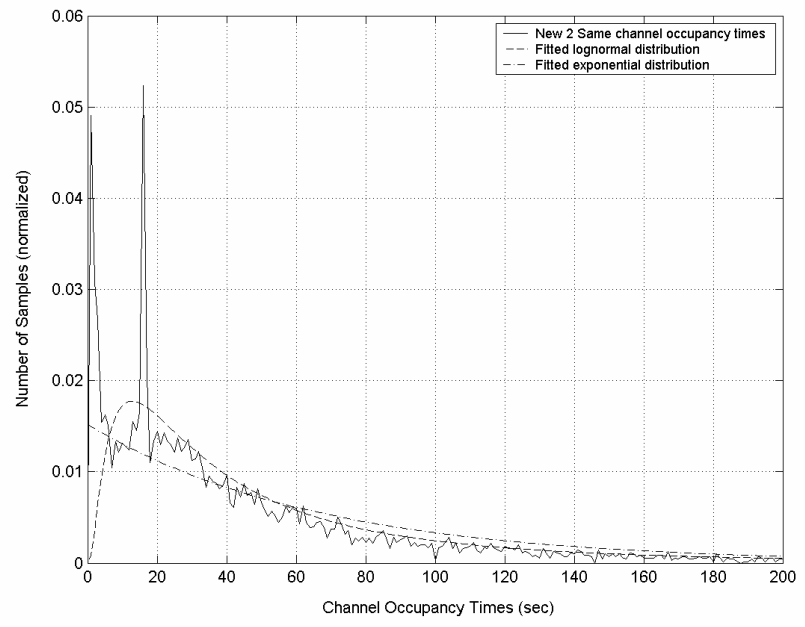

Fig. 1. Distribution of channel occupancy times (new2same) and the fitted lognormal and exponential distributions.

new2sameorho and total and the spikes observed around the $4^{\text {th }}$ second within channel occupancy time samples classified as ho2sameorho are due to same samples that created the spikes within channel occupancy time samples classified as new2same and ho2ho, respectively.

\section{Statistical TOOLS}

We choose the following statistical distributions that can reasonably represent the observed empirical data: exponential distribution, lognormal distribution, gamma distribution and weibull distribution. Other distributions such as Beta, Poisson or Pareto are not proposed as candidate distributions in this study due to lack of theoretical and empirical criteria to support them as candidates.

\section{A. Parameter Estimation}

The parameters for each candidate distribution can be estimated from the empirical data set using methods such as probability plotting, method of moments and maximum likelihood estimation (MLE). We prefer to use MLE, which selects a value as an estimate for which the observed sample is most likely to occur, due to its consistency and efficiency under certain regularity conditions when the sample size approaches infinity [17]. Besides, it gives better fit results than others when the goodness of fit test is applied [18].

\section{B. Goodness-of-fit-tests}

Goodness-of-fit tests are statistical hypothesis tests that determine whether a specific statistical distribution is appropriate to characterize an observed sample data set [19]. However, failure to reject the null hypothesis that claims the observed data samples to be IID random variables with a particular distribution function, should not be interpreted as accepting the null hypothesis as being true. Law and Kelton noted in [18] that these tests are often not very powerful for small to moderate sample sizes and thus should be regarded as a systematic approach for detecting fairly gross differences

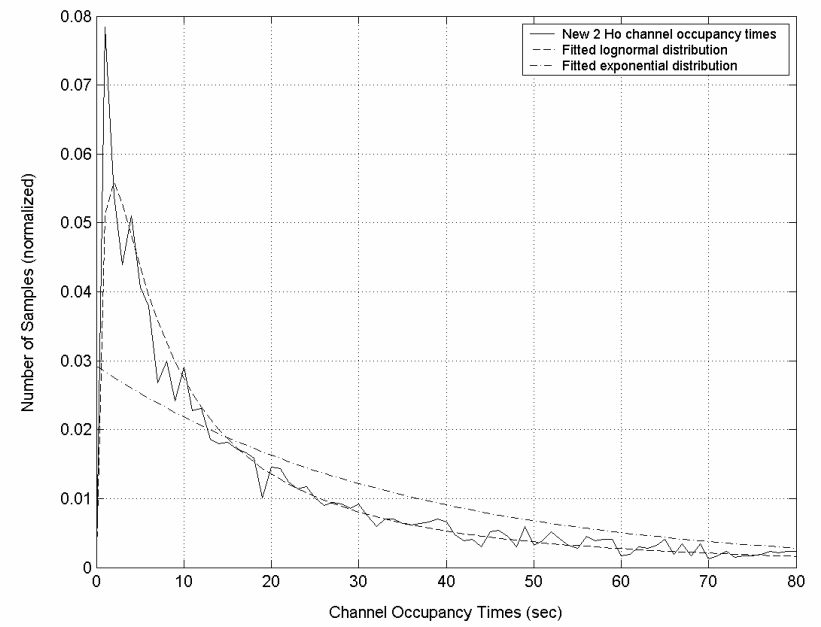

Fig. 2. Distribution of channel occupancy times (new2ho) and the fitted lognormal and exponential distributions.

instead. Yet if the sample size is very large, the authors observed that these tests almost always reject the null hypothesis. Therefore it is more important to find the statistical distribution that best fits the empirical data even if it may be rejected with a "relatively small" margin. In this paper, we are not only looking for a distribution that is accepted by the test, but also the order in which the candidate distributions fit the empirical data set since it is usually sufficient to have a distribution that is "nearly" correct due to its benefits.

The chi-square test is the oldest goodness-of-fit hypothesis test that can be thought of as a more formal comparison of a histogram with the proposed candidate statiscial distribution. To compute the chi-square test statistic in either continuous or discrete case, the entire range of the candidate distribution must be divided into $k$ adjacent intervals $\left[\mathrm{t}_{0}, \mathrm{t}_{1}\right),\left[\mathrm{t}_{1}, \mathrm{t}_{2}\right), \ldots,\left[\mathrm{t}_{\mathrm{k}-1}, \mathrm{t}_{\mathrm{k}}\right)$, where $t_{0}$ and $t_{k}$ can either or both be $-\infty$ and $+\infty$, respectively. Then we check the number of observations in the $j$ th interval $\left[\mathrm{t}_{\mathrm{k}-1}, \mathrm{t}_{\mathrm{k}}\right), \mathrm{N}_{\mathrm{j}}$, for $j=1,2, \ldots, k$ and compute the expected proportion $p_{j}$ of the observations that would fall in the $j$ th interval if we were sampling from the candidate statistical distribution. In the continuous case,

$$
p_{j}=\int_{t_{j-1}}^{t_{j}} f_{c}(x) \cdot d x
$$

where $f_{c}(\mathrm{x})$ is the probability density function of the continuous candidate distribution. For discrete data,

$$
p_{j}=\sum_{t_{j-1} \leq x_{i}<t_{j}} f_{d}\left(x_{i}\right)
$$

where $f_{d}\left(x_{\mathrm{i}}\right)$ is the probability distribution function of the discrete candidate distribution. Finally, the test statistic is

$$
\chi^{2}=\sum_{j=1}^{k} \frac{\left(N_{j}-n p_{j}\right)^{2}}{n p_{j}}
$$




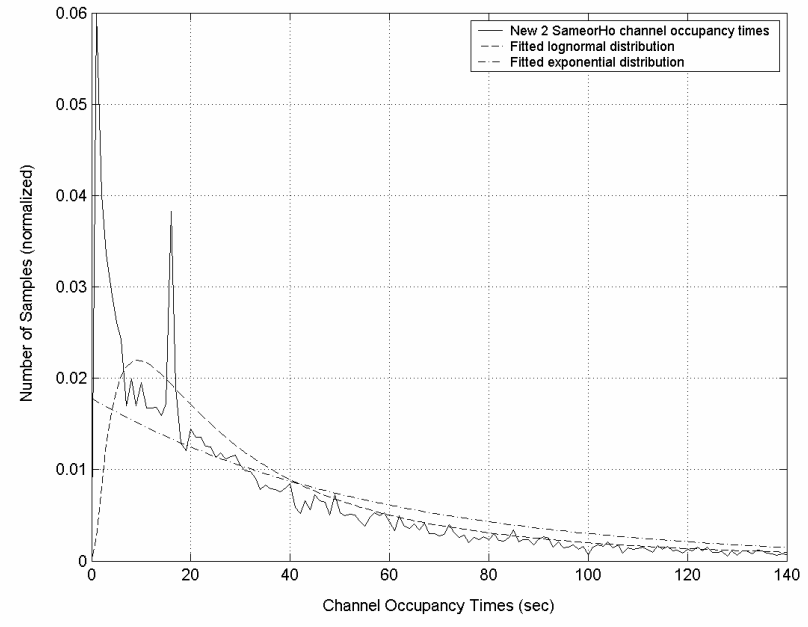

Fig. 3. Distribution of channel occupancy times (new2sameorho) and the fitted lognormal and exponential distributions.

The test statistic is expected to be small if the fit was good since $n p_{j}$ will then be the expected number of observations that would fall in the $j$-th interval. The most troublesome aspect of carrying out a chi-square goodness of fit test is choosing the number and size of the bins. This is a difficult problem and no definitive prescription can be given that is guaranteed to produce good results in terms of validity and high power for all hypothesized distributions and all sample sizes. However Law and Kelton suggested a few guidelines in [17]. The authors proposed the equiprobable approach which chooses the bin intervals so that the expected proportion of the empirical data set that fall in each interval will be equal to each other.

Kolmogorov-Smirnov (K-S) tests, on the other hand, compare an empirical data set with a candidate distribution without grouping the data and thus eliminating the troublesome problem of interval specification. These tests are valid for any sample size and only if all the parameters of the hypothesized distribution are known and the distribution is continuous. However, the range of applicability is more limited than that for chi-square tests [18].

In this paper, we use the chi-square test which remains in wide use since it can be applied to any hypothesized distribution with parameters estimated from the observed data. We perform the following steps:

1. Divide the data into groups with respect to occupancy types and sort it by channel occupancy times.

2. Choose a list of candidate distributions to which each group of data will be fitted and calculate the parameters for each distribution using the corresponding MLE equations.

3. Choose the number of bins into which each group of data will be divided, calculate the expected number of observations in each bin, and confirm that it exceeds the minimum number of expected number of observations given in [18]. For each group of data, the bin boundaries should satisfy

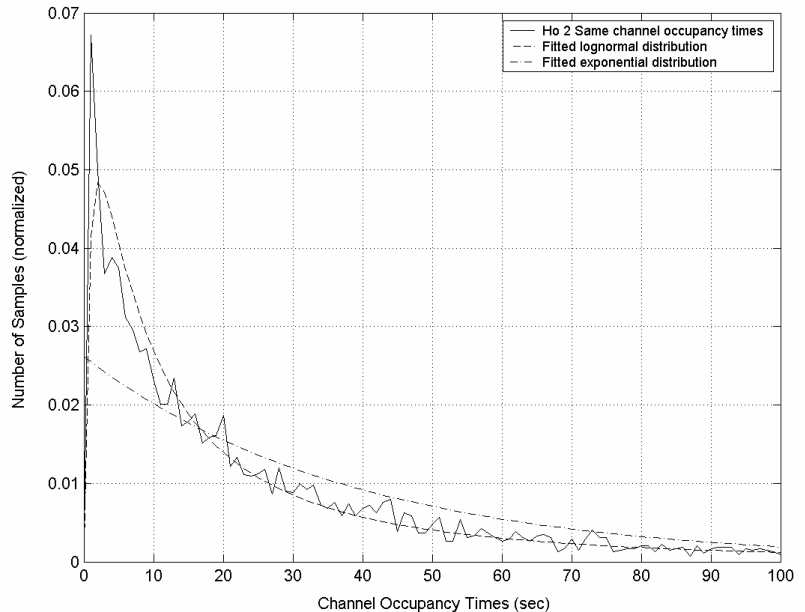

Fig. 4. Distribution of channel occupancy times (ho2same) and the fitted lognormal and exponential distributions.

$$
\left\lfloor n p_{j}=n \cdot\left(p r\left\{X<t_{j}\right\}-p r\left\{X<t_{(j-1)}\right\}\right) \mid \geq 5\right.
$$

where $j=1,2, \ldots, k$,

$p_{j}$ is the probability of a data item that falls into bin $j$,

$k$ is the number of bins,

$t_{0}, t_{1}, \ldots t_{k}$ are the upper bin boundaries,

$n$ is the total number of observations in the data set and $n p_{j}$ is the expected number of observations in the $j$-th bin.

4. Divide the observed data in each group into its corresponding created bins. Each data group should be made continuous by spreading it evenly within 0.5 seconds of their discrete values.

5. Calculate the test statistics for each group using (3).

6. The null hypothesis is rejected in each case if the value of the test statistics is greater than the value of the chi-square statistics with $k-1-z$ degrees of freedom, where $z$ is the number of parameters estimated. We set the significance level of the performed tests equal to $0.95(\alpha=0.05)$.

\section{NumERicAl Results}

\section{A. Channel Occupancy Times}

In this section, we present the statistical results obtained from the goodness-of-fit tests performed for each aforementioned type of channel occupancy times. The results show that all types of channel occupancy times resemble either lognormal or weibull distributions. However only for two of these, new2ho and ho2new, the candidate probability distributions were able to pass the chi-square goodness-of-fit test with lognormal being a better fit than weibull. None of the remaining channel occupancy times fits statistically to a proposed candidate distribution due to the observed anomalies. Thus, filtering these empirical data sets is inevitable since no candidate distribution can otherwise be fitted to the data. 


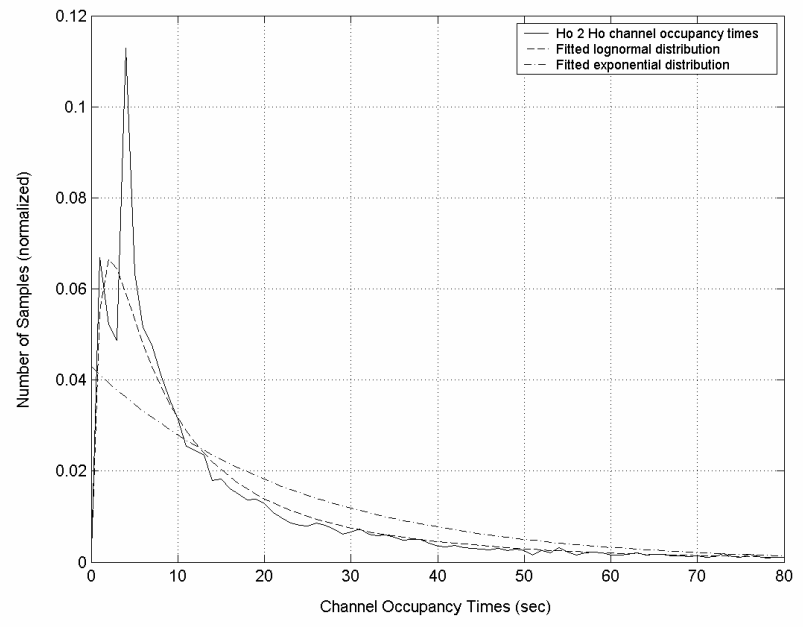

Fig. 5. Distribution of channel occupancy times (ho2ho) and the fitted lognormal and exponential distributions.

We discard all short channel occupancy times less than 3 seconds from the respective data sets of channel occupancy time distributions classified as new2same, new2sameorho and total since most of them are calls terminated abnormally as previously mentioned. The excess channel occupancy times observed around $16^{\text {th }}$ second in the same data sets given above and around $4^{\text {th }}$ second in the data sets of channel occupancy time distributions classified as ho2ho and ho2sameorho are stripped from the rest using simple means. The revised goodness-of-fit test results and the chi-square statistics for each type of channel occupancy are not presented here due to space constraints but a summary of the results is as follows: Lognormal distribution passes the chi-square goodness-of-fit tests for channel occupancy types of new2same, new 2 ho, ho2same and total along with weibull distribution which is the second best fit except for new2same. For channel occupancy types of new2sameorho, ho2ho and ho2sameorho, lognormal distribution does not pass the chi-square goodness-of-fit test, yet it appears to be the best fit due to its little margin with the chi-square statistics. Figs. 1 to 7 show the fitted lognormal and exponential distribution along with the normalized histograms for all types of channel occupancy times, respectively.

\section{B. Call Holding Times for Stationary and Mobile Users}

In this section, we present the statistical results obtained from the goodness-of-fit tests performed to fit a proposed candidate distribution to channel occupancy and call holding times of stationary and mobile users. In [12] and [13], the authors demonstrated theoretically that traffic characteristics such as channel occupancy times depend not only on call holding times but also on users' mobility which can be characterized by cell residence times. However it is difficult to obtain cell residence times since the observed data is collected from network nodes which track idle users infrequently. Thus, we classify users in our data set based on the number of handoffs that they commit. We identify each user with zero

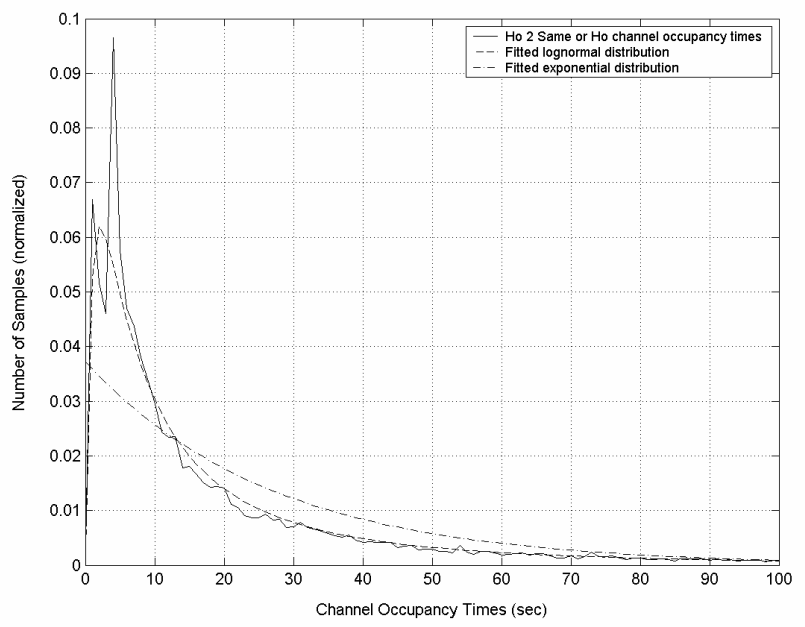

Fig. 6. Distribution of channel occupancy times (ho2sameorho) and the fitted lognormal and exponential distributions.

number of handoffs stationary or low mobility and the rest mobile. Note that some stationary users may in fact be physically mobile within a cell yet we still consider them stationary with respect to their cell residency. We assume users mobile if the number of handoffs that they commit is more than a certain threshold (set to 3 handoffs in this study) since "pilot pollution" may cause a stationary call to commit handoff once in a while.

We consider users affiliated with new2same stationary whereas users affiliated with new2ho, new2sameorho, ho2same, ho2ho and ho2sameorho mobile. Hence, call holding time distribution for stationary users is equivalent to the channel occupancy time distribution for new2same. However we have to obtain the call holding time distribution for mobile users (total mobility) separately since the distribution for total includes times affiliated with both stationary and mobile users. The results for all channel occupancy types are previously given. We apply the chi-square goodness-of-fit test to total_mobility after the observed anomalies were stripped using simple means. Fig. 8 shows the fitted lognormal distribution, along with the normalized histogram for mobile users' call holding times. In [14], Fang et al., showed analytically that when cell residence times are exponentially distributed, channel occupancy time distribution for "new calls" can be approximated by the fitted exponential distribution for stationary users (or when mobility is low) and yet for mobile users there is a significant mismatch between channel occupancy time distribution for "handoff calls" and the fitted exponential distribution. We observe that not only call holding and channel occupancy times of mobile users fit lognormal distribution very strongly compared to others but also both distributions for stationary users fit the lognormal distribution while radically differing from the fitted exponential distribution. Hence we expect cell residence times not to be exponentially distributed. 


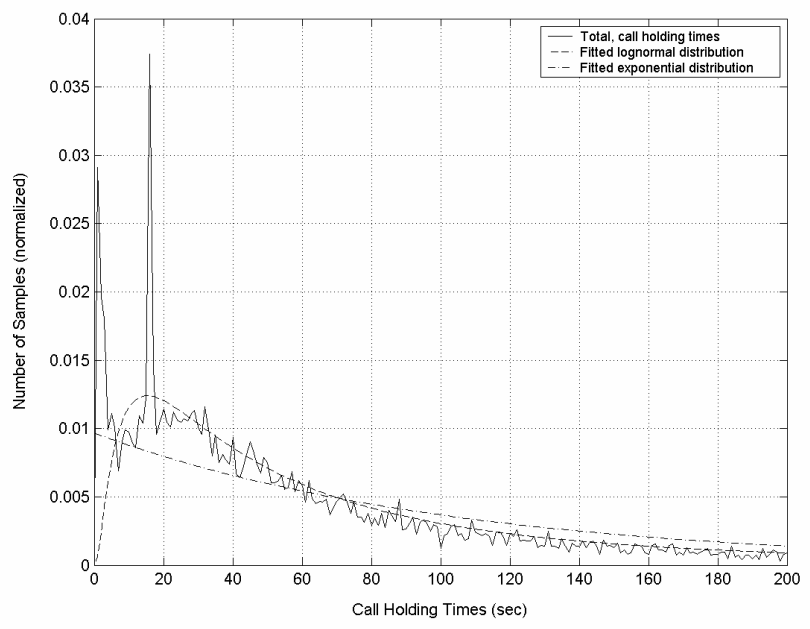

Fig. 7. Distribution of call holding times (total) and the fitted lognormal and exponential distributions.

\section{CONCLUSION}

In classical voice traffic modeling call holding times are approximated by exponential distributions. However, in cellular networks, channel occupancy time distributions are needed instead for analytical system modeling or to feed simulations. In this paper, we classify channel occupancy times and present an empirical study to determine which probability distribution functions can approximate them better. Although the results are environment dependent, we have made no assumptions that can influence the results as opposed to previous analytical and simulation studies. For stationary users, channel occupancy and call holding times have previously been approximated by exponential distributions due to their tractability, assuming that cell residence times are exponentially distributed. However we have shown that lognormal distribution fits much better to not only all types of channel occupancy times but also call holding times for stationary and mobile users. The results are expected to be useful in traffic and network modeling, performance evaluation, billing, network management and optimization.

\section{REFERENCES}

[1] D. Hong and S. S. Rappaport, "Traffic model and performance analysis for cellular mobile radiotelephone systems with prioritized and nonprioritized handoff procedures," IEEE Transactions on Vehicular Technology, vol. 35, pp. 77-92, Aug. 1986.

[2] R. Ramjee, R. Nagarajan, and D. Towsley, "On optimal call admission control in cellular networks," Wireless Networks, vol. 3, no. 1, pp. 2941, March 1997.

[3] Y. Fang, and Y. Zhang, "Call admission control schemes and performance analysis in wireless mobile networks," IEEE Transactions on Vehicular Technology, vol. 51, no.2, pp. 371-382, March 2002.

[4] M. Naghshineh and S. Schwartz, "Distributed call admission control in mobile/wireless networks," IEEE Journal on Selected Areas in Communications, vol. 14, no. 4, pp.711-717, May 1996.

[5] A. Gersht and K. J. Lee, "A bandwidth management strategy in ATM networks," Technical report, GTE Laboratories, 1990.

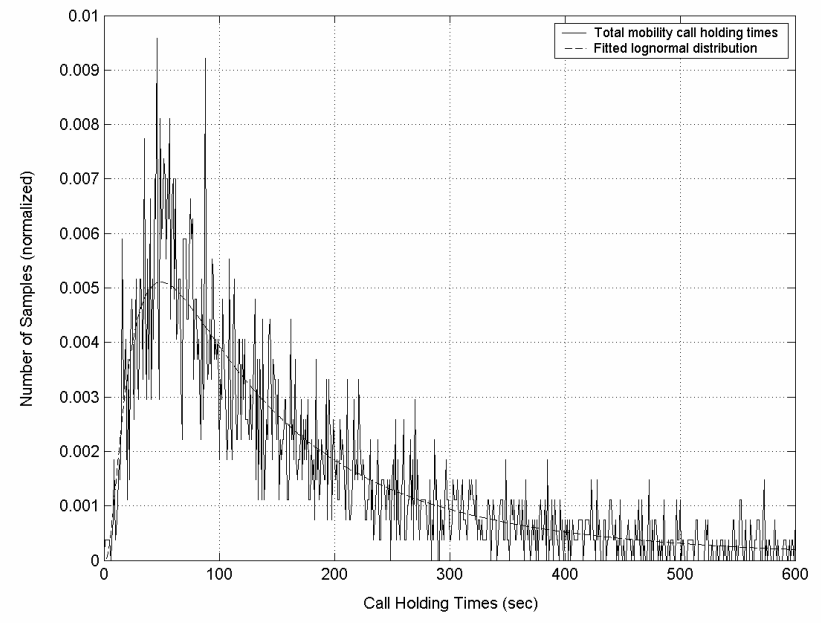

Fig. 8. Distribution of call holding times (total_mobility) and the fitted lognormal distribution.

[6] S. C. Borst and D. Mitra, "Virtual partitioning for robust resource sharing: computational techniques for heterogeneous traffic," IEEE Journal on Selected Areas in Communications, vol. 16, no. 5, pp. 668678, June 1998.

[7] E. A. Yavuz and V. C. M. Leung, "Computationally Efficient Method to Evaluate the Performance of Guard-Channel-Based Call Admission Control in Cellular Networks," IEEE Transactions on Vehicular Technology, vol. 55, no. 4, pp. 1412-1424, July 2006.

[8] S. S. Rappaport, "The multiple call handoff problem in personal communications networks," IEEE 40th Vehicular Technology Conference, pp. 287-294, May 1990.

[9] S. S. Rappaport and G. Monte, "Blocking, hand-off and traffic performance for cellular communication systems with mixed platforms," IEEE 42nd Vehicular Technology Conference, vol. 2, pp. 1018-1021, May 1992.

[10] R. A. Guerin, "Channel Occupancy Time Distribution in a Cellular Radio System," IEEE Transactions Vehicular Technology, vol. 35, no. 3, pp. 89-99, 1987.

[11] C. Jedrzycki, and V. C. M. Leung, "Probability distribution of channel holding time in cellular telephony systems", IEEE Vehicular Technology Conference (VTC'96), vol. 1,pp. 247 - 251, Apr. 1996.

[12] Y. Fang, I. Chlamtac, and Y.B. Lin, "Call performance for a PCS networks", IEEE Journal on Selected Areas in Communications vol. 15, no. 8, pp. 1568-1581, Oct. 1997.

[13] Y. Fang, I. Chlamtac, and Y.B. Lin, "Modeling PCS networks under general call holding times and cell residence time distributions", IEEE Transactions on Networking vol. 5,no. 6, pp. 893 - 906, Dec. 1997

[14] Y. Fang, I. Chlamtac and Y.B. Lin, "Channel occupancy times and handoff rate for PCS networks", IEEE Transactions on Computers vol. 47, no. 6, pp. $679-692$, June 1998.

[15] F. Barcelo, and J. Jordan, "Channel Holding Time Distribution in Public Telephony Systems (PAMR and PCS)", IEEE Transactions on Vehicular Technology, vol. 49, no. 5, pp. 1615 - 1625, Sep. 2000.

[16] V. A. Bolotin, "Modeling Call Holding Time Distributions for CCS Network Design and Performance Analysis", IEEE Journal on Selected Areas in Communications, vol. 12, no.3, pp. 433 - 438, April 1994.

[17] K. S. Trivedi, Probability and Statistics with Reliability, Queuing and Computer Science Applications, New Jersey: Prentice-Hall, 1982.

[18] A. M. Law and W. D. Kelton, Simulation, Modeling and Analysis, 3rd edition, New York:McGraw-Hill, 2000.

[19] M. R. Sheldon, Introduction to Probability and Statistics for Engineers and Scientists, 3rd edition, USA: Elsevier Academic Press, 2004. 